\title{
Extreme galactic wind and star formation at low and high redshift
}

\author{
S. Lípari ${ }^{1,2}$, Y. Taniguchi ${ }^{3,2}$, E. Mediavilla ${ }^{4,2}$, H. Dottori ${ }^{5}$, R. \\ Terlevich $^{6}$, M. Ajiki ${ }^{3,2}$, Y. Shioya ${ }^{3}$, R. J. Diaz ${ }^{1,2}$, B. Garcia-Lorenzo ${ }^{4,2}$, \\ J. Acosta-Pulido ${ }^{4}$ and W. Zheng ${ }^{7,2}$ \\ ${ }^{1}$ Cordoba Observatory and CONICET, Laprida 854, 5000 Cordoba, Argentina \\ ${ }^{2}$ Visiting astronomer at GEMINI, SUBARU, KECK II, ESO, LA PALMA, BALEGRE, \\ CASLEO Observatories \\ ${ }^{3}$ Astronomical Institute, Tohoku University, Aoba, Sendai 980-8578, Japan \\ ${ }^{4}$ Instituto de Astrofisica de Canarias, 38205 La Laguna, Tenerife, Spain \\ ${ }^{5}$ Instituto de Fisica, Univ. F. R. Grande do Sul, CP 15051, P. Alegre, Brazil \\ ${ }^{6}$ Institute of Astronomy, Madingley Road, Cambridge CB3 0HA, United Kingdom \\ ${ }^{7}$ Department of Physics \& Astronomy, Univ. of Johns Hopkins, Baltimore, USA
}

\begin{abstract}
From a long-term study of IR mergers/QSOs (at low redshift), we report detailed spectroscopic evidences for outflow (OF)and starburst features. We found extreme velocity $\mathrm{OF}$ (EVOF) in IRAS 01003-2238, 11119+3257, 13218+0552, 14394+5332, 15130-1958 and 154620450. The EVOF were detected mainly in objects with strong starburst plus obscured IR QSOs. Meanwhile, the low velocity OF components were detected in objects with starburst and LINER processes. HST images of IR+BAL+Fe II QSOs show in practically all of these objects "arc or shell" features probably associated to galactic-winds. In addition, results from a study of twodimensional (2D) fibre spectroscopy of IR mergers/QSOs with galactic winds are presented. Comparing our data base for IR mergers/QSOs with two sample of nearby mergers we found in both samples a high proportion (75 per cent) of IR mergers with galactic winds.

From a very deep imaging and spectroscopic survey of Ly $\alpha$ emitters (LAEs) at $\mathrm{z}=5.7$ we present the results of a study of sample of 20 LAEs candidates. Two of these objects have been already confirmed as LAE at $\mathrm{z}=5.7$. We detected in one of these LAE (at $\mathrm{z}=5.687$ ), spectral features probably associated with the more distant galactic wind observed to date.
\end{abstract}

\section{Introduction}

One of the most important astrophysical issues in modern astronomy is to explore the formation and early evolution of galaxies at high redshift. More specifically, the understanding of galaxy formation is one of our main goals in the astrophysics. In order to improve the understanding of this issue, it is necessary to study: (I) at low redshif, object with strong star formation and galactic winds (GW) processes, specially IR mergers/QSOs (Lipari et al. 2003, 2004); (II) at high redshift, a well-defined sample of such forming galaxies and then investigate their nature unambiguously (e.g., Ajiki et al. 2002, 2003). Motivated by this, our team began an international observational Proyect on intensive investigations of star-forming + GW galaxies since 1999 (Lipari et al. 2003, 2004).

\section{Galactic Winds and Star Formation at Low Redshift}

The first step for this Proyect is to understand the star formation process in nearby galaxies because we can obtain a lot of unambiguous information because they are bright 
enough to be observed in detail. In particular, we study mainly IR mergers/QSOSs, which are excellent laboratory to analyze nearby extreme star formation processes and GW.

We presented in this section mainly a summary of our results obtained from optical spectroscopy and high resolution HST-WFPC2 broad band images, of selected IR mergers and QSOs. These results can be summarized as follows:

(1) Detailed kinematical and/or morphological evidences for OF and WR features were found, in the nearby IR mergers NGC 4038/39, IRAS 23128-5919 (with low velocity OF), and in the nearby QSOs IRAS 01003-2238, 11119+3257, 13218+0552, 14394+5332, 15130-1958 and 15462-0450 (with extreme velocity OF). We also detected probably OF in IRAS 05024-1941, 13305-1739, 13451+1232 and 23389+0300.

We found that the low velocity OF components were detected mainly in objects with starburst processes, i.e., OF associated to galactic-winds generated in multiple type II SN explosions and massive stars. Meanwhile the EVOF were detected mainly in objects with strong starburst plus obscured IR QSOs.

(2) HST images of IR+BAL+Fe II QSOs show in practically all of these objects "arc or shell" features probably associated to galactic-winds (i.e., to multiple type II SN explosions, or to starburst+AGN) and/or merger processes.

(3) Recently we have obtained 2D high resolution spectroscopy for NGC 2623, Arp 220, Mrk 231, and others IR systems (at La Palma, WHT) in order to include in our study new 2D maps of the kinematic, emission line and continuum (Lipari et al. 2004). These maps will help to study in detail these type of arcs: those associated to outflowing "shocked" material and to tidal loops/tails in galactic collisions.

(4) Comparing our data base for IR mergers/QSOs with two sample of nearby mergers we found in both samples a high proportion (75 per cent) of IR mergers with galactic winds. These results suggest that: (a) GWs are frequent event in IR mergers and probably in IR QSOs, (b) extreme starburst+GW and extreme IR emission could be simultaneous processes induced by merger events. This latter conclusion is also supported by a clear trend found in the plot OF velocity vs. $\log$ Lir, in the sense that extreme OF velocities are detected only in extreme IR emitters (Lipari et al. 2004).

\section{Galactic Winds and Star Formation at High Redshift}

In the second program, we have conducted our own very deep imaging survey for Lyman $\alpha$ emitters at $\mathrm{z}=5.7 \mathrm{using}$ the prime-focus camera (Suprime-Cam) on the Subaru Telescope (Ajiki et al. 2002, 2003). This survey gave us a well-defined photometric sample of $20 \mathrm{LAE}$ candidates at $\mathrm{z}=5.7$ and two of them have been already confirmed as LAEs (Ajiki et al. 2002, Taniguchi et al. 2003). Recently, we obtained deep R images at Gemini South (in this area) for GMOS multi-spectroscopy. Ajiki et al. (2002) found in the optical Keck II spectrum of J1044-0130 (at $\mathrm{z}=5.687$ ) the typical line profile of galactic wind. Thus this object present probably the more distant GW observed to date.

\section{References}

Ajiki, M., et al. 2002, ApJ, 576, L25

Ajiki, M., et al. 2003, AJ, 126, 2091

Lipari, S., et al. 2003, MNRAS, 340, 289

Lipari, S., et al. 2004, MNRAS, 348, 369

Taniguchi, Y., et al. 2003, ApJ, 585, L97 